\title{
Predictors of Successful Fast Track Protocol in Transfemoral Transcatheter Aortic Valve Implantation Under General Anaesthesia
}

Tamer Owais ${ }^{1,2^{*}}$, Iliazbek Kazakbaev ${ }^{1}$, Mohammad El Garhy ${ }^{1,3}$, Norbert Salewsky ${ }^{4}$, Lars Hüter ${ }^{4}$, Torsten Schreiber ${ }^{4}$, Waheedullah Karzai ${ }^{4}$ and Thomas Kuntze $^{1}$ ${ }^{1}$ Department of Cardiac Surgery, Heart Centre, Zentralklinik Bad Berka, Germany

${ }^{2}$ Department of Cardiothoracic Surgery, Cairo University, Egypt

${ }^{3}$ Department of Cardiology, Minia University, Egypt

${ }^{4}$ Department of Anesthesia and Intensive Care Medicine, Zentralklinik Bad Berka, Germany

*Corresponding author: Tamer Owais, Department of Cardiac Surgery, Heart Centre, Zentralklinik Bad Berka, Robert-Koch Allee 9 , 99437 Bad Berka, Germany, Tel: 493418650; E-mail: tamerowaiss1976@yahoo.com

Received: March 06, 2018; Accepted: March 16, 2018; Published: March 20, 2018

Copyright: @ 2018 Owais T, et al. This is an open-access article distributed under the terms of the creative commons attribution license, which permits unrestricted use, distribution, and reproduction in any medium, provided the original author and source are credited.

\begin{abstract}
Objectives: There is no consensus about the optimal length of hospital stay in patients undergoing elective transfemoral TAVR under general anesthesia. Our study tried to find predictors of failure of early discharge strategy in those patients.

Methods and results: We included retrospectively 135 high risk TAVI patients (mean Log. EuroSCORE $30.8 \pm 6$ ) operated under general anaesthesia between June 2017 and January 2018 in central hospital, Bad Berka, Germany. Focus on the preoperative factors was established in favor to find possible predictors of longer hospital stay in this group of patients. Uni- and multivariate analysis were performed to identify independent preoperative predictors of fast-track protocol failure, defined as inability to discharge the patient from the hospital five days after surgery. Independent predictors of fast-track protocol failure were NYHA IV (OR 4.3; CI 95\% 2.3-25, p-Value 0.017), ejection fraction (EF) $\leq 30 \%$ (OR 3.5; Cl 95\% 2-12.5, p-Value 0.006), right bundle branch block (OR 4.7; Cl 95\% 1.25-25, pValue 0.03 ) and stroke with residual disability (OR 4.5; CI 95\% 1.1-16, p-Value 0.025).
\end{abstract}

Conclusion: NYHA IV, severely reduced ejection fraction, right bundle branch block and stroke with residual disability might be considered potential predictors of failure of fast track protocol in patient undergoing TAVI under general anesthesia.

Keywords: TAVI; Fast track; Transfemoral

\section{Introduction}

We were enthusiastic to do this study because there exists no consensus on the optimal length of stay in patients undergoing elective transfemoral TAVR under general anaethesia, and this issue is not addressed in guideline statements. Fast track protocol has been previously published, under local anaesthesia successfully reducing the postoperative stay to 3 days [1-3]. Moreover, successful fast track protocol in transapical TAVI under general anaesthesia has been implemented and published as a single centric prospective study [4].

We report here our experience with Fast track TAVI under general anaesthesia but with 5 postoperative days. Comparatively, some studies report the mean hospital stay following TAVI to be 11-13 days $[5,6]$, while other contemporary studies demonstrate hospitalization duration shorter than three days in approximately $30 \%$ of patients [1-3].

Due to the intensive TAVI diagnostic workup and the complexity of the prosthesis and procedure, TAVI became an extremely resource intensive procedure $[5,6]$. The economical profit should not be the only concern, but also reducing infection, improving psychological status as well as optimizing rehabilitation. We aimed in this study to report our experience with fast-track protocol of patients undergoing transcatheter aortic valve implantation under general anaesthesia and to identify preoperative predictors, which may influence the success of the fast-track protocol.

\section{Methods}

We conducted a retrospective study for patients operated between June 2017 and January 2018. One hundred thirty-five consecutive high-risk patients (mean age 80.2 years \pm 4.1 years, mean Log. EuroSCORE $30.8 \pm 6$ ) underwent TAVI in our institute. This time frame was chosen after we reached more than 1500 TAVI patients in our institute which were all done under general anaesthesia in order to overcome the learning curve associated-complications. The decision whether the fast track was to be applied or not was met through a multidisciplinary meeting of the heart team, consisting of 1 cardiac surgeon, 1 interventional cardiologists and 1 anaesthesiologists. We assessed all TAVI candidates according to our defined fast track protocol which was designed based on regular recommendations, guidelines and previously published papers in this domain which defines specific exclusion criteria (renal dialysis, pulmonary oedema and cardiogenic shock) to find out the best option for each patient. We investigated the results after implementation of this protocol in our institute with concentrated focus on the clinical variables that may predict fast-track protocol failure. Patient's data were collected retrospectively to record more than 40 different clinical variables 
Citation: Owais T, Kazakbaev I, El Garhy M, Salewsky N, Hüter L, et al. (2018) Predictors of Successful Fast Track Protocol in Transfemoral Transcatheter Aortic Valve Implantation Under General Anaesthesia. J Cardiovasc Dis Diagn 6: 310. doi:10.4172/2329-9517.1000310

Page 2 of 6

(Tables 1-3). Uni- and multivariate analysis were performed to identify independent preoperative predictors of fast-track protocol failure, is defined as inability to discharge the patient from the hospital five days after surgery. All patients signed an informed detailed consent.

\begin{tabular}{|c|c|c|c|c|}
\hline \multirow[t]{2}{*}{ Variables } & \multirow{2}{*}{$\begin{array}{l}\text { Total } \\
\mathrm{N}=135\end{array}$} & \multirow{2}{*}{$\begin{array}{l}\text { Fast Track } \\
\mathrm{N}=80\end{array}$} & \multirow{2}{*}{$\begin{array}{l}\text { Slow Track } \\
\mathrm{N}=55\end{array}$} & \multirow[t]{2}{*}{ p-Value } \\
\hline & & & & \\
\hline Age (years $\pm S D$ ) & $80.2 \pm 4.1$ & $80.50 \pm 5.303$ & $79.84 \pm 5.962$ & 0.4 \\
\hline Male n (\%) & $76(56.2 \%)$ & $49(61 \%)$ & $27(49 \%)$ & 0.2 \\
\hline COPD n (\%) & $32(23.7 \%)$ & $16(20 \%)$ & $16(29 \%)$ & 0.3 \\
\hline iCA Stenosis unilateral n (\%) & $14(10.3 \%)$ & $8(10 \%)$ & $6(10.9 \%)$ & 1 \\
\hline iCA Stenosis bilateral n (\%) & $8(5.9 \%)$ & $5(6.3 \%)$ & $3(5.4 \%)$ & 1 \\
\hline iCA intervention n (\%) & $11(8.1 \%)$ & $6(7.5 \%)$ & $5(9.1 \%)$ & 0.7 \\
\hline Stroke without residual n (\%) & $14(10.3 \%)$ & $7(8.8 \%)$ & $7(12.7 \%)$ & 0.5 \\
\hline Stroke with residual n (\%) & $6(4.4 \%)$ & $1(1.3 \%$ & $5(9.1 \%)$ & 0.04 \\
\hline Dementia n (\%) & $3(2.2 \%)$ & $1(1.8 \%)$ & $2(3.6 \%)$ & 0.5 \\
\hline Redo n (\%) & $18(13.3 \%)$ & $10(12.5 \%)$ & $8(14.5 \%)$ & 0.7 \\
\hline PTCA n (\%) & $57(42.2 \%)$ & $34(42.5 \%)$ & $23(41.8 \%)$ & 1 \\
\hline CRF >II n (\%) & $47(34.8 \%)$ & $28(35 \%)$ & $19(34.5 \%)$ & 1 \\
\hline $\operatorname{ccs} 4 \mathrm{n}(\%)$ & $32(23.7 \%)$ & $20(25 \%)$ & $12(21.8 \%)$ & 0.8 \\
\hline NYHA IV n (\%) & $80(59.2 \%)$ & $42(52.4 \%)$ & $38(69.1 \%)$ & 0.039 \\
\hline Previous myocardial infarction n (\%) & $37(27.4 \%)$ & $23(28.7 \%)$ & $14(25.5 \%)$ & 0.7 \\
\hline $\mathrm{PH}$ n (\%) & $74(54.8 \%)$ & $42(53.2 \%)$ & $32(58.2 \%)$ & 0.6 \\
\hline Valvuloplasty n (\%) & $5(3.7)$ & $2(2,5 \%)$ & $3(5.5 \%)$ & 0.39 \\
\hline PVD n (\%) & $18(13.3)$ & $10(12.5 \%)$ & $8(14.5 \%)$ & 0.79 \\
\hline DM Insulin n (\%) & $28(20.7 \%)$ & $15(18.8 \%)$ & $13(23.6 \%)$ & 0.5 \\
\hline BMI>30 n (\%) & $26(19.2 \%)$ & $19(23.8 \%)$ & $7(12.7 \%)$ & 0.12 \\
\hline Mobility n (\%) & $125(92.5 \%)$ & $73(91 \%)$ & $52(94.5 \%)$ & 0.5 \\
\hline $\mathrm{Hb}$ (mmol/l) (preoperative) & $8 \pm 0.9$ & $8 \pm 1.0$ & $8 \pm 0.8$ & 1 \\
\hline Corticosteroid therapy n (\%) & $10(7.4 \%)$ & $4(6 \%)$ & $6(10.9 \%)$ & 0.3 \\
\hline Tumor n (\%) & $28(20.7 \%)$ & $19(23.8 \%)$ & $9(16.4 \%)$ & 0.3 \\
\hline Log EuroScore (mean $\pm \mathrm{SD})$ & $30.8 \pm 6$ & $30.5 \pm 7$ & $31.4 \pm 4$ & 0.1 \\
\hline STS (mean \pm SD) & $10.9 \pm 3$ & $10.4 \pm 2$ & $11.7 \pm 3$ & 0.8 \\
\hline
\end{tabular}

Table 1: Comparison between the groups as regard baseline characteristics.

Our cohort group of patients were subjected to an Intensive care unit stay of 2 days then directly transferred to the normal ward on telemetry bed; if clinically stable; until the fourth day. Discharge to the rehabilitation hospitals or home took place on the fifth day. Early mobilization and physiotherapy began on the $1^{\text {st }}$ postoperative day. Trans-thoracic echocardiography was done on the second day before discharge from the ICU to rule out pericardial effusion. Electrocardiography was done on daily bases until discharge.

Routine TAVI work up was routinely done including electrocardiography, trans-esophageal echocardiography, coronary angiography, carotid duplex as well as computed tomography (CT) aortography until the peripheral arterial tree. The aortic valve annulus 
Citation: Owais T, Kazakbaev I, El Garhy M, Salewsky N, Hüter L, et al. (2018) Predictors of Successful Fast Track Protocol in Transfemoral Transcatheter Aortic Valve Implantation Under General Anaesthesia. J Cardiovasc Dis Diagn 6: 310. doi:10.4172/2329-9517.1000310

Page 3 of 6

was sized using three-dimensional (3D) trans-esophageal

echocardiography and CT.

\begin{tabular}{|c|c|c|c|c|}
\hline \multirow{2}{*}{ Variables } & Total & Fast Track & Slow Track & \multirow{2}{*}{$p$-Value } \\
\hline & $N=135$ & $N=80$ & $N=55$ & \\
\hline Pacemaker n (\%) & $23(17 \%)$ & $19(23 \%)$ & $4(7.3 \%)$ & 0.01 \\
\hline AF n (\%) & $61(45.1 \%)$ & $30(37.5 \%)$ & $31(56.5 \%)$ & 0.03 \\
\hline LBBB n (\%) & $19(14 \%)$ & $10(12.5 \%)$ & $9(16.4 \%)$ & 0.6 \\
\hline RBBB n (\%) & $9(6.6 \%)$ & $2(2.5 \%)$ & $7(12.7 \%)$ & 0.003 \\
\hline$E F<30 \%$ n (\%) & $20(14.8 \%)$ & $9(11.4 \%)$ & $11(20 \%)$ & 0.04 \\
\hline Ml>II n (\%) & $27(20 \%)$ & $15(18.8 \%)$ & $12(21.8 \%)$ & 0.6 \\
\hline TI >II n (\%) & $25(18.5 \%)$ & $14(17.5 \%)$ & $11(20 \%)$ & 0.8 \\
\hline$M G A V m m H g \pm S D$ & $49.6 \pm 16$ & $50.1 \pm 14.6$ & $49 \pm 20$ & 0.4 \\
\hline
\end{tabular}

Table 2: Comparison between the groups as regard preoperative echocardiographic and electrocardiographic finding.

\begin{tabular}{|c|c|c|c|c|}
\hline \multirow[t]{2}{*}{ Variables } & Total & Fast Track & Slow Track & \multirow[t]{2}{*}{ p-Value } \\
\hline & $\mathrm{N}=135$ & $\mathrm{~N}=80$ & $\mathrm{~N}=55$ & \\
\hline Creatinine $(\mu \mathrm{mol} / \mathrm{l})$ (1. pop.d.) & $92.6 \pm 44.2$ & $85.6 \pm 37$ & $97.9 \pm 53$ & 0.1 \\
\hline Pericardial effusion & $16(11.8 \%)$ & $9(11.3 \%)$ & $7(12.7 \%)$ & 0.2 \\
\hline Vascular complication & $7(5.1 \%)$ & $2(2.5 \%)$ & $5(9.1 \%)$ & 0.09 \\
\hline Sternotomy & $3(2.2 \%)$ & 0 & $3(5.4 \%)$ & N.A. \\
\hline $\mathrm{AVB} \geq 2^{\circ}$ & $10(7.4 \%)$ & $6(7.5 \%)$ & $4(7.3 \%)$ & 1 \\
\hline Cereberal insult & $5(3.7 \%)$ & 0 & $4(7.3 \%)$ & N.A. \\
\hline Inotropic therapy & $60(44.4 \%)$ & $31(39.2 \%)$ & $29(52.7 \%)$ & 0.08 \\
\hline Inotropes $>6$ hours & $33(24.4 \%)$ & $13(16.3 \%)$ & $20(36.4 \%)$ & 0.04 \\
\hline Ventilatory time post-operative (minutes) & $24 \pm 120$ & $12 \pm 132$ & $36 \pm 198$ & 0.3 \\
\hline Confusion & $23(17 \%)$ & $11(13.7 \%)$ & $12(21.8 \%)$ & 0.06 \\
\hline Transfusion (within 24 h pop.) & $16(11.8 \%)$ & $7(8.9 \%)$ & $9(16.4 \%)$ & 0.2 \\
\hline 30-day Mortality & $1(0.7 \%)$ & $1(1.2 \%)$ & 0 & N.A. \\
\hline 30-day Hospital readmission & $2(1.4 \%)$ & $1(1.2 \%)$ & $1(1.8 \%)$ & 0.2 \\
\hline
\end{tabular}

Table 3: Comparison between the groups as regard clinical end points.

Elective TAVI patients were all admitted on the day before the procedure, based on our institutional practice. All procedures were performed under fluoroscopic guidance, in a standard Hybrid room with surgical backup under general anaesthesia. The transcatheter heart valves implanted included Edwards SAPIEN, SAPIEN XT and SAPIEN 3, Symetis ACURATE TA and neo THVs. All arterial sheaths were removed with closure devices at the end of the procedure. In case of no evidence of acute intraprocedural conduction abnormalities (severe bradycardia $<45 \mathrm{bpm}$, second-degree Atrio-ventricular block or third-degree Atrio-ventricularblock), the temporary pacemaker was maintained in situ and removed in the intensive care unit only after electrophysiological consultation to exclude the indication of a permanent pacemaker. Pacemaker implantation was indicated if there is persistence of an atrioventricular block (Mobitz II or III) more than 24 hours after TAVI. Patients were eligible for early discharge if the procedure and the postoperative phase were free of major 
Page 4 of 6

complications. We set the rule of "never insist on continuing on fast track at the expense of patient's safety".

According to the length of hospital stay, cases were classified into fast track ( $\leq 5$ days) and slow track ( $>5$ days). We defined length of stay as the days elapsed between the operative day of TAVI until the date of discharge. We did not include the preoperative period into the length of stay as our study focused only on patients that were not in an acute shock phase. We considered patients safety as the first priority and thus 30 days safety was followed up and recorded. It was defined as absence of re-admission and mortality up to 30 days. Clinical followup was performed through office visits and telephone contacts.

\section{Statistical analysis}

Continuous variables are expressed as means and standard deviations. Parametric and non-parametric tests were used based on sample size and normality of distribution. Categorical data are expressed as percentages. Univariable analysis using Fisher exact and chi square were used to identify differences between successful and failed fast track protocol groups. In order to determine the factors associated with failed fast track protocol, logistic regression model was generated. All analysis was done using SPSS statistical software (IBM Corp. Released 2013. IBM SPSS Statistics for Windows, Version 22.0. Armonk, NY: IBM Corp).

\section{Results}

Our retrospective results show the following: one hundred forty-six (146) TAVIs were performed during the study period, 11 patients were excluded from this study (7.5\%). One Patient excluded because of intraoperative mortality and 10 patients had one of the abovementioned exclusion criteria. from the remaining 135 patients, 80 were discharged within five days of the index procedure ('fast track group' $80 / 135,59 \%)$. 30-day mortality was $0.7 \%$, one patient from fast track group died in the $28^{\text {th }}$ postoperative day due to unknown cause. 30 day hospital readmission was $1.4 \%$ without significant difference between the both groups and was due to hypertensive crisis in one patient in the slow tract group and one patient in the fast tract group due to non-cardiac cause (femoral fracture). Vascular complications and postoperative blood transfusion were more prevalent in slow track group but without significant difference, may be due to small number of patients $(2.5 \%$ vs $9.1 \%$ p-Value $=0.09,8.9 \%$ vs $16.4 \%$ p-Value 0.2$)$. We recorded in this study an overall high percentage of catecholamine use $44.4 \%$ at $0 \mathrm{~h}$ postoperative and $24.4 \% 6 \mathrm{~h}$ postoperative with statistically significant difference in the $6 \mathrm{~h}$ variable between the two groups. Fast-track protocol failure (37\%) was due to vascular complications in 5 patients $(9.1 \%)$, cerebral insult 4 patients $(7.3 \%)$, need of catecholamine therapy postoperative $>6$ hours in 17 patients (30.1\%), 3 patients (6\%) were sternotomised, 12 patients $(21.9 \%)$ due to confusion, 7 patients $(12.8 \%)$ due to pericardial effusion, progressively rising creatinine in 4 patients $(7.3 \%)$ and 3 patients $(5.5 \%)$ due to respiratory insufficiency. There was no difference between the early discharge and late discharge group in terms of 30days hospital readmission and mortality (Table 3).

The independent predictors of fast-track protocol failure were NYHA IV (OR 4.3; CI 95\% 2.3-25, p-Value 0.017), ejection fraction $(\mathrm{EF}) \leq 30 \%$ (OR 3.5; CI 95\% 2-12.5, p-Value 0.006), right bundle branch block (OR 4.7; CI 95\% 1.25-25, p-Value 0.03) and stroke with residual disability (OR 4.5; CI 95\% 1.1-16, p-Value 0.025) (Table 4).

\begin{tabular}{|l|l|l|l|l|}
\hline \multirow{2}{*}{ Variables } & \multicolumn{4}{l}{$95.0 \%$ CI for B } \\
\cline { 2 - 5 } & OR & Lower & Upper & Sig. \\
\hline Stroke with residual & 4.5 & 1.1 & 16 & 0.025 \\
\hline NYHA IV & 4.3 & 2.3 & 25 & 0.017 \\
\hline EF<30\% & 3.5 & 2 & 12.5 & 0.006 \\
\hline RBB & 4.7 & 1.25 & 25 & 0.03 \\
\hline
\end{tabular}

NYHA: New York Heart Association; EF: Ejection Fraction; RBBB: Right Bundle Branch Block.

Table 4: Predictors of fast track failure in multivariate analysis.

\section{Discussion}

The application of a fast track protocol in TAVI was not only targeting the economical aspect; which is limiting the expansion of this procedure; or minimizing the unnecessary use of medical resources but also the desire to accelerate patient's recovery and mobilization. Economical target could be an objective but has to be only reached without compromising the patients or procedures safety. In the PARTNER study, postprocedural length of hospital stay was 7.4 and 12.4 days for transfemoral and transapical TAVI, respectively [7].

We stick to the total postoperative stay of 5 days after general anaesthesia despite of the presence of many other published studies which sticks for 3 days as a fast track protocol after local anaesthesia. We support our point of view by the overcoming the liable phase of conduction abnormalities postoperatively which was evident in the first 5 postoperative days [8]. Moreover, the previous trial to implement a fast track protocol in patient undergoing TAVI under general anaesthesia; but with a comparable patients' risk profile with our study; showed a successful shortening of ICU stay in "fast track group" but with average hospital stay of 10 days [4]. However, this trial was in transapical TAVI patients which associated with longer hospital stay than transfemoral approach [9].

Before we implemented fast track protocol in our institution we had asked ourselves two questions; whether the mortality and morbidity of TAVI in the early discharge group could have been reduced by keeping these patients in hospital for longer period? And what if we achieved a reasonable outcome; can we expand our inclusion criteria in future?

We found out that application of this fast track protocol in patients underwent TAVI under general anaesthesia was not associated with increased mortality or morbidity in comparisons with previous studies, 30 -day mortality was $0.7 \%$ vs $4.4 \%$ in Durand et al [1]. This coincides with Barbanti and Aldalati in their studies [2,3]. It's worth noting that we have an extra intraoperative mortality which we excluded from our statistics because we it was not related to the implementation of the fast track protocol.

Our 30 -days readmission rate is $(1.4 \%)$. In addition, there is no significant difference in the readmission rate in the 2 groups and this supports the safety of fast track protocol application and the noninferiority of fast track under general anaesthesia because our readmission rate is lower than what's is published in other series. It's worth mentioning that the only readmission in the fast track group was due to femoral fracture and this could have been excluded from our statistical groups being a non-cardiac cause and hence could have lowered our general readmission rate $[1,2]$. 
Our vascular complications rate was $5.1 \%$ which is coinciding with the data published in other series [1-4]. We are proud to keep this rate in spite of the high prevalence of PVD (peripheral vascular disease) in our patients $(13.3 \%)$ and the introduction of a new closure device in august 2017. The introduction of the new closure device could have influenced our results negatively due to the expected learning curve associated-complications. We consider our centre a high volume centre with a good considerably experienced team and this explains the relatively low incidence of vascular complications.

Neither vascular complications nor blood transfusion were an independent predictor of sloping out of our fast track group in our study. This could be interpreted through the previous paragraph. Blood transfusion variables have been previously studied after TAVI and interesting results have been published by Barbanti et al. [10] and Durand et al. [1]. The former demonstrated that lack of significant bleeding is a predictor of early discharge and the later demonstrated that blood transfusion post-TAVI was a negative predictor of successful fast track.

We recorded in this study an overall high percentage of catecholamine use $44.4 \%$ at $0 \mathrm{~h}$ postoperative and $24.4 \% 6 \mathrm{~h}$ postoperative with statistically significant difference in the $6 \mathrm{~h}$ variable between the two cohort groups. Our general higher consumption rate of catecholamine could be explained by the negative inotropic effect of anaesthetic agent, routine use of balloon valvuloplasty and rapid pacing. In addition, we believe that catecholamine therapy was necessary to achieve a sufficient perfusion pressure especially in elderly patients with cerebrovascular insufficiency i.e., it was sometimes not intended for the sake of cardiac support.

Surprisingly the success rate of fast track protocol was 59\% despite the use of general anaesthesia in such high-risk patients. We still operate most of the intermediate risk patients conventionally, so we included in this study a higher portion of higher risk patients (Log EuroScore $30.8 \%)$ in comparison to other studies $(16.9 \% \pm 9.6 \%$ Durand et al. and 20.8\% Aldalati et al). After Partner II study there is a general slow approach towards intermediate risk groups and it's as well reasonable to assume that the indications for TAVI will expand into intermediate and even lower risk groups [11]. Consequently, this protocol may be utilized more frequently in the future.

Concerning the independent predictors of fast-track protocol failure, we concluded that NYHA IV, ejection fraction $(\mathrm{EF}) \leq 30 \%$, atrial fibrillation, right bundle branch block and stroke with residual disability were accused. This coincides with Papadopoulos et al., who identified $\mathrm{EF} \leq 30$ and others as independent factors of failure [4]. We oppose each other in mitral regurge, as it was not found to be an independent factor of failure in our cohort group and we think that this could be explained by the difference between his approach as transapical (burden on left ventricle through the apical ventriculotomy approach) and ours as transfemoral. Arbel et al. [9] in his multicentric retrospective study showed that low EF, high NYHA and atrial fibrillation were associated with prolonged hospital stay and this agrees with our results. We believe this supports our theory of being noninferior as all of our patients were operated under general anaesthesia while his study groups were operated under local and general anaesthesia. Astonishingly, Right bundle branch block was found to be one of our predictors while it was not the case in Barbanti et al. and Durand et al. After revising our preoperative ECG database for comparative purposes, we should confess that 6 out of the 7 patients in the slow track group had bifasicular block which could be responsible for this result.
We are glad to reach this limited mortality, morbidity, readmission and success rate of 5-days hospital stay despite our study was implemented under general anaesthesia. Other studies as Duran and Arbel stated that TAVI under conscious sedation is a predictor of early discharge rate further [1,9]; nonetheless, we can consider our results as non-inferior and satisfactory. We limited our practice only to fast track but under general anaesthesia in spite of being capable of performing it under local anaesthesia. This is because we needed to go through this new protocol in our institute gradually without patient's compromise. It would be worth to consider in future switching to local anaesthesia practice.

\section{Study Limitations}

Finally, the limitations of our study are: first being a retrospective observational study, and there may have residual confounders that we did not account for. Second: we overpassed few clinical parameters in our study as wound infection and/or dehiscence and familial social situation. We can consider our study as definite non-conclusive study but may be hypothesis productive. Moreover, our study was not a randomized trial and consequently it could be considered a nonpowerful study and cannot conclude an optimal length of stay for patients undergoing elective transfemoral TAVR. We believe that there is a necessity of more prospective studies to evaluate the optimal length of hospital stay postoperatively after TAVI.

\section{Conclusion}

NYHA IV, severely reduced ejection fraction, right bundle branch block and stroke with residual disability might be considered potential predictors of failure of fast track protocol in patient undergoing TAVI under general anesthesia.

\section{References}

1. Durand E, Eltchaninoff H, Canville A, Bouhzam N, Godin M, et al. (2015) Feasibility and safety of early discharge after transfemoral transcatheter aortic valve implantation with the Edwards SAPIEN-XT prosthesis. Am J Cardiol 115: 1116-1122.

2. Aldalati O, Keshavarzi F, Kaura A, Byrne J, Eskandari M, et al. (2018) Factors associated with safe early discharge after transcatheter aortic valve implantation. Cardiology J 25: 14-23.

3. Papadopoulos N, El-Sayed Ahmad A, Thudt M, Fichtlscherer S, Meybohm P, et al. (2016) Successful fast track protocol implementation for patients undergoing transapical transcatheter aortic valve implantation. J Cardiothorac Surg 11: 55.

4. Babaliaros V, Devireddy C, Lerakis S, Leonardi R, Iturra SA, et al. (2014) Comparison of transfemoral transcatheter aortic valve replacement performed in the catheterization laboratory (minimalist approach) versus hybrid operating room (standard approach): outcomes and cost analysis. JACC Cardiovasc Interv 7: 898-904.

5. Reinohl J, Kaier K, Gutmann A, Sorg S, von Zur MC, et al. (2015) Inhospital resource utilization in surgical and transcatheter aortic valve replacement. BMC Cardiovasc Disord 15: 132.

6. Reynolds MR, Magnuson EA, Wang K, Lei Y, Vilain K, et al. (2012) PARTNER Investigators. Cost-effectiveness of transcatheter aortic valve replacement compared with surgical aortic valve replacement in high-risk patients with severe aortic stenosis: results of the PARTNER (Placement of Aortic Transcatheter Valves) trial (Cohort A). J Am Coll Cardiol 60: 2683-2692.

7. Khawaja MZ, Rajani R, Cook A, Khavandi A, Moynagh A, et al. (2011) Permanent pacemaker insertion after CoreValve transcatheter aortic valve implantation: incidence and contributing factors (the UK CoreValve Collaborative). Circulation 123: 951-960. 
Citation: Owais T, Kazakbaev I, El Garhy M, Salewsky N, Hüter L, et al. (2018) Predictors of Successful Fast Track Protocol in Transfemoral Transcatheter Aortic Valve Implantation Under General Anaesthesia. J Cardiovasc Dis Diagn 6: 310. doi:10.4172/2329-9517.1000310

Page 6 of 6

8. Arbel Y, Zivkovic N, Mehta D, Radhakrishnan S, Fremes SE, et al. (2017) Factors associated with length of stay following trans-catheter aortic valve replacement - a multicenter study. BMC Cardiovasc Disord 17: 137

9. Barbanti M, Capranzano P, Ohno Y, Attizzani GF, Gulino S, et al. (2015) Early discharge after transfemoral transcatheter aortic valve implantation. Heart 101: 1485-1490.
10. Leon MB, Smith CR, Mack MJ, Makkar RR, Svensson LG, et al. (2016) Transcatheter or surgical aortic-valve replacement in intermediate-risk patients. N Engl J Med 374: 1609-1620.

11. Abawi M, Nijhoff F, Agostoni P, Emmelot-Vonk MH, de Vries R (2016) Incidence, predictive factors, and effect of delirium after transcatheter aortic valve replacement. JACC Cardiovasc Interv 9: 160-168. 